\title{
Density-of-states Monte Carlo method for simulation of fluids
}

\author{
Qiliang Yan, Roland Faller, and Juan J. de Pabloa) \\ Department of Chemical Engineering, University of Wisconsin-Madison, Madison, Wisconsin 53706
}

(Received 6 November 2001; accepted 30 January 2002)

\begin{abstract}
A Monte Carlo method based on a density-of-states sampling is proposed for study of arbitrary statistical mechanical ensembles in a continuum. A random walk in the two-dimensional space of particle number and energy is used to estimate the density of states of the system; this density of states is continuously updated as the random walk visits individual states. The validity and usefulness of the method are demonstrated by applying it to the simulation of a Lennard-Jones fluid. Results for its thermodynamic properties, including the vapor-liquid phase coexistence curve, are shown to be in good agreement with high-accuracy literature data. (c) 2002 American Institute of Physics. [DOI: 10.1063/1.1463055]
\end{abstract}

\section{INTRODUCTION}

The free energy landscapes of complex systems, such as proteins or glasses, are characterized by the existence of deep, local free energy minima. These minima pose significant obstacles for molecular simulations; once a system is trapped in a minimum, conventional algorithms are unable to explore configurations pertaining to other, relevant regions of phase space. Several Monte Carlo methodologies have been developed in the last decade to circumvent the sampling problem. Examples include expanded ensembles, ${ }^{1,2}$ multicanonical algorithms, ${ }^{3}$ and parallel tempering formalisms. ${ }^{4}$ All of these techniques have improved considerably our ability to simulate the equilibrium properties and structure of complex materials, including polymers, proteins in solution, or organic glasses.

Most of these techniques have relied on the Metropolis et al. original prescription, ${ }^{5}$ in which trial configurations of a system are accepted or rejected according to probability distributions pertaining to conventional statistical mechanical ensembles (or minor alterations thereof). A notable exception is provided by multicanonical methods, in which trial configurations are accepted according to multicanonical weights constructed in such a way as to "flatten out" high energy barriers between distinct configurations. Unfortunately, the required multicanonical weights must be determined through a tedious and highly computationally demanding iterative process. In multicanonical methods as well as in more conventional Monte Carlo techniques for molecular simulation, the condition of detailed balance is satisfied by construction.

Recently, however, Wang, and Landau ${ }^{6}$ proposed a promising approach for lattice systems that eliminates some of the shortcomings of the original multicanonical prescription. The key to that approach is to accept trial configurations of the system according to a running estimate of the density of states; by design, their formalism does not strictly enforce detailed balance. A random walk in energy space is used to evenly visit each energy level. The density of states of an energy level is modified by an arbitrary convergence factor

${ }^{\text {a)} E l e c t r o n i c ~ m a i l: ~ d e p a b l o @ e n g r . w i s c . e d u ~}$ when that energy level is visited. By controlling that factor in a systematic manner, these authors were able to generate the density of states of an Ising lattice system to high accuracy in a self-consistent way. We refer to this approach as the density-of-states (DOS) Monte Carlo method.

For simulations on a lattice, the DOS Monte Carlo method promises to offer significant advantages over previously available techniques. It is therefore of interest to explore whether analogous ideas can be used in a continuum, in the context of a realistic fluid. Several challenges must be overcome: first, random moves on a lattice system can only give rise to a small set of discrete energy changes, thereby simplifying considerably the nature of the simulations. Random displacements in a continuum result in unpredictable energy changes, and it is unclear whether the DOS Monte Carlo method can be implemented at all. Second, and perhaps more important, the DOS Monte Carlo method generates an estimate of the density of states to within a multiplicative constant. Such a constant depends on the particular length of a simulation, on system size (volume), and on density (number of particles). On a lattice, it is common practice to study systems of constant composition. Furthermore, the system is generally assumed to be incompressible. Real fluids, however, are not incompressible, and their study over any reasonable range of density would require that the absolute density of states (and its multiplicative constant) be known. A naive extension of the lattice DOS method to a real fluid would therefore require that multiple simulations be conducted at different densities, and that the resulting densities of states corresponding to each calculation be matched with each other in some fashion to estimate each multiplicative constant. This procedure would be prone to uncertainties related to the manner in which different thermodynamic states are combined, it would be time consuming, and it would not offer significant advantages over thermodynamic integration or histogram reweighting. ${ }^{\text {? }}$

In this work we propose a DOS formulation that addresses these issues. Its implementation is demonstrated in the context of a Lennard-Jones fluid, for which highaccuracy thermodynamic data area available. It is shown that the proposed simulation technique is able to generate the 
partition function of the system over a wide range of energy and density, which comprises an infinitely dilute gas and a dense liquid. This is achieved within a single simulation, at a small fraction of the computational demands of currently available simulation techniques.

\section{DENSITY OF STATES MONTE CARLO METHOD}

On a lattice, a DOS Monte Carlo method generates a random walk in energy space by flipping spins in a random manner. A trial flip is accepted according to criteria that eventually result in a flat distribution of energy. Generating a flat energy distribution requires that microscopic states having internal energy $E$ be visited with a probability inversely proportional to the density of states, $g(E)$. Accepting trial spin flips with probability

$$
p\left(E_{1} \rightarrow E_{2}\right)=\min \left[1, \frac{g\left(E_{1}\right)}{g\left(E_{2}\right)}\right]
$$

(where $E_{1}$ and $E_{2}$ are the energies before and after the spin is flipped) would therefore result in a flat energy distribution.

Unfortunately, the density of states $g(E)$ is not known $a$ priori. The central idea in the DOS Monte Carlo method is to construct the density of states on the fly. At the beginning of a simulation, a constant density of states is assumed for all energy levels. As the simulation proceeds, the instantaneous values of $g(E)$ are used to accept trial flips. Every time that an energy level $E$ is visited, the density of states for that level is modified according to $g(E) \rightarrow g(E) * f$, where $f>1$ is an arbitrary convergence factor. An energy histogram $H(E)$ is accumulated during the course of the simulation. When that histogram becomes sufficiently flat, the convergence factor is reduced to a finer value, e.g., $f_{i+1}=\sqrt{f_{i}}$; the histogram $H(E)$ is reset to zero, and the simulation is continued. This process is repeated until the convergence factor $f$ becomes arbitrarily small [say, less than $\left.\exp \left(10^{-8}\right)\right]$.

In the many-body fluid formulation proposed here, the number of particles or molecules in the system is allowed to fluctuate. We consider a phase space characterized by internal energy $E$ and number of particles $N$. To sample relevant regions of phase space efficiently, our strategy is to have each pair of $(N, E)$ points be visited uniformly. In other words, a microscopic state having $N$ particles and energy level $E$ should be visited with probability $1 / g(N, E)$, where $g(N, E)$ represents the two-dimensional degeneracy of $(N, E)$ states.

For simplicity, only two types of trial moves are considered. The first type of move consists of simple trial displacements, in which the coordinates of a randomly chosen particle are altered by a small random amount. A trial move is then accepted or rejected according to

$$
p\left(E_{1}, N \rightarrow E_{2}, N\right)=\min \left[1, \frac{g\left(E_{1}, N\right)}{g\left(E_{2}, N\right)}\right] .
$$

The second type of move consists of trial insertions or destruction of particles. For a trial insertion, a particle is introduced into the system at a random position. This move is accepted with probability

$$
p(N \rightarrow N+1)=\min \left[1, \frac{V}{(N+1) \Lambda^{3}} \frac{g\left(N, E^{\text {old }}\right)}{g\left(N+1, E^{\text {new }}\right)}\right],
$$

where $V$ is the volume of the system, and $\Lambda$ is the de Broglie thermal wavelength. In Eq. (3), $E^{\text {old }}$ and $E^{\text {new }}$ represent the energies of the system before and after a particle is introduced, respectively, and $g\left(N, E^{\text {old }}\right)$ and $g\left(N+1, E^{\text {new }}\right)$ are the corresponding densities of states. For a trial destruction, a randomly chosen particle is removed from the system, and the move is accepted with probability:

$$
p(N \rightarrow N-1)=\min \left[1, \frac{N \Lambda^{3}}{V} \frac{g\left(N, E^{\mathrm{old}}\right)}{g\left(N-1, E^{\mathrm{new}}\right)}\right] .
$$

It is of interest to remark that traditional, Metropolistype simulation techniques penalize trial insertions or destructions of particles by a factor proportional to the exponential of the energy, $\exp (-\beta \Delta E)$, where $\Delta E$ represents the change in energy created by the trial move, and where $\beta$ $=1 / k_{\mathrm{B}} T$ ( $T$ is the temperature). If $\Delta E$ exceeds a few $k_{\mathrm{B}} T$, the trial move is generally rejected. That factor is absent from the algorithm proposed in this work, thereby facilitating considerably the creation or destruction of molecules and the sampling of high-density configurations.

A running estimate of the two-dimensional density of states is continuously updated as the simulation proceeds. When a configuration having $N$ particles and energy $E$ is visited, the current value of $g(N, E)$ is multiplied by a convergence factor $f$. A two-dimensional histogram of number of particles and energy $H(N, E)$ is constructed. When that histogram is deemed to be sufficiently flat, the histogram is discarded and the simulation is continued, this time with a smaller convergence factor.

Having generated a density of states according to the procedure outlined above, a partition function for the system can be constructed at any given temperature and chemical potential according to

$$
\Xi(T, \mu)=\sum_{N} \sum_{E} g(N, E) e^{-\beta E+N \beta \mu} .
$$

The partition function determined from Eq. (5) is only known to within an arbitrary constant multiplier. Our simulation, however, comprises the special case $N=0$; the density of states for that case is known to be unity, thereby providing a means to the determine the absolute value of $\Xi$ for all other states. Thermodynamic properties of interest can subsequently be determined from knowledge of $\Xi(T, \mu)$. For example, the thermodynamic pressure and the thermodynamic internal energy can be calculated according to

$$
p(T, \mu)=\frac{k_{\mathrm{B}} T}{V} \log \Xi(T, \mu)
$$

and

$$
U(T, \mu)=\frac{\sum_{N} \sum_{E} E g(N, E) e^{-\beta E+N \beta \mu}}{\sum_{N} \sum_{E} g(N, E) e^{-\beta E+N \beta \mu}} .
$$


The compressibility of the fluid can also be inferred from $g(N, E)$ by considering fluctuations in the number of particles according to

$$
\kappa(T, \mu)=-\frac{1}{V}\left(\frac{\partial V}{\partial p}\right)_{N, T}=\frac{V}{k_{\mathrm{B}} T} \frac{\left\langle N^{2}\right\rangle-\langle N\rangle^{2}}{\langle N\rangle^{2}},
$$

where

$$
\langle N\rangle=\frac{\sum_{N} \sum_{E} N g(N, E) e^{-\beta E+N \beta \mu}}{\sum_{N} \sum_{E} g(N, E) e^{-\beta E+N \beta \mu}}
$$

and

$$
\left\langle N^{2}\right\rangle=\frac{\sum_{N} \sum_{E} N^{2} g(N, E) e^{-\beta E+N \beta \mu}}{\sum_{N} \sum_{E} g(N, E) e^{-\beta E+N \beta \mu}} .
$$

In many applications, it is of particular interest to determine the precise location of first or second order thermodynamic phase transitions. In the particular case of a simple fluid, to calculate the liquid-vapor binodal curve, a simple two-state construction can be used. At a temperature well below the critical point, the equilibrium density distribution exhibits two distinct peaks; a threshold number of particles $N_{0}$ can be designated, such that all $N \leqslant N_{0}$ states can be regarded as pertaining to a "vapor" branch, and all $N>N_{0}$ states as belonging to a "liquid" branch. The pressures corresponding to these two branches can be calculated according to

$$
\begin{aligned}
& p^{V}(T, \mu)=\frac{k_{\mathrm{B}} T}{V} \log \sum_{N \leqslant N_{0}} \sum_{E} g(N, E) e^{-\beta E+N \beta \mu,} \\
& p^{L}(T, \mu)=\frac{k_{\mathrm{B}} T}{V} \log \sum_{N>N_{0}} \sum_{E} g(N, E) e^{-\beta E+N \beta \mu} .
\end{aligned}
$$

For any given temperature, a phase coexistence point can be found by carefully tuning the value of chemical potential $\mu_{0}$ in such a way as to satisfy the condition $p^{V}\left(T, \mu_{0}\right)=p^{L}\left(T, \mu_{0}\right)$. The coexistence densities of the vapor and liquid phases can then be calculated as

$$
\begin{array}{r}
\rho^{V}(T)=\frac{\sum_{N \leqslant N_{0}} \sum_{E} N g(N, E) e^{-\beta E+N \beta \mu_{0}}}{V \sum_{N \leqslant N_{0}} \sum_{E} g(N, E) e^{-\beta E+N \beta \mu_{0}},} \\
\rho^{L}(T)=\frac{\sum_{N>N_{0}} \sum_{E} N g(N, E) e^{-\beta E+N \beta \mu_{0}}}{V \sum_{N>N_{0}} \sum_{E} g(N, E) e^{-\beta E+N \beta \mu_{0}}} .
\end{array}
$$

Note that the equilibrium pressure obtained from Eqs. (11) and (12) differs from that obtained from Eq. (6) by a term $-k_{\mathrm{B}} T \log 2 / V$, which arises from finite-size effects. For systems having a large enough volume, however, this difference is negligible.
One particular problem that must be addressed in the method proposed above is that the relevant range of energy is strongly dependent on the number of particles in the system. The energy levels accessible to a two-particle system are different than those accessible to a 100-particle system. To determine energy ranges for different system sizes, two short preliminary DOS simulations are run, one at the lowest temperature, and the other at the highest temperature. The goal of these simulations is to achieve a flat distribution of $N$, as opposed to trying to make the $(N, E)$ histogram flat; the energy distribution is dictated by a conventional Boltzmann weight. A flat $N$ distribution would be obtained if each microscopic state with $N$ particles was visited with probability $1 / Q(N)$, where $Q(N)$ is the canonical partition function (of a system having $N$ particles).

The scheme followed in these preliminary runs is similar to that adopted above: a table of partition functions is constructed, with entries for each value of $N$. At the beginning of a simulation, the partition functions are set to unity for all entries of $N$. Two types of moves are used: particle displacements, accepted according to conventional Metropolis criteria, and particle insertion or destruction moves, accepted with probability

$$
\begin{aligned}
p(N \rightarrow N+1)= & \min \left[1, \frac{V}{(N+1) \Lambda^{3}} \frac{Q(N)}{Q(N+1)}\right. \\
& \times \exp (-\beta \Delta E)]
\end{aligned}
$$

and

$$
p(N \rightarrow N-1)=\min \left[1, \frac{N \Lambda^{3}}{V} \frac{Q(N)}{Q(N-1)} \exp (-\beta \Delta E)\right],
$$

where $\Delta E$ is the energy change associated with the trial particle insertion or destruction. Upon each visit to a microscopic state, the corresponding $Q(N)$ is updated by multiplying it by an arbitrary convergence factor $f$. The minimum and maximum values of energy corresponding to each number of particles are tracked during the simulation; the minimum energy at the lowest temperature and the maximum energy of the highest temperature define the relevant energy range to be used in the two-dimensional $g(N, E)$ DOS production simulations.

Since the purpose of the preliminary runs is to determine the energy range, it is not necessary to generate a perfectly flat histogram. The only requirement is that each number of particles be visited with enough frequency. It is important to emphasize, however, that the scheme described above for $Q(N)$ is very useful on its own right, for example, for calculation of the chemical potential of a fluid. If this DOS $Q(N)$ simulation is run until convergence is achieved (i.e., until the $N$ histograms are flat and the convergence factor $f$ is small enough), the result is the free energy of the system as a function of $N$. This information is particularly useful in expanded ensemble simulations, ${ }^{1,2}$ where the weights associated with individual expanded states (which are closely related to free energies) must be determined before a produc- 


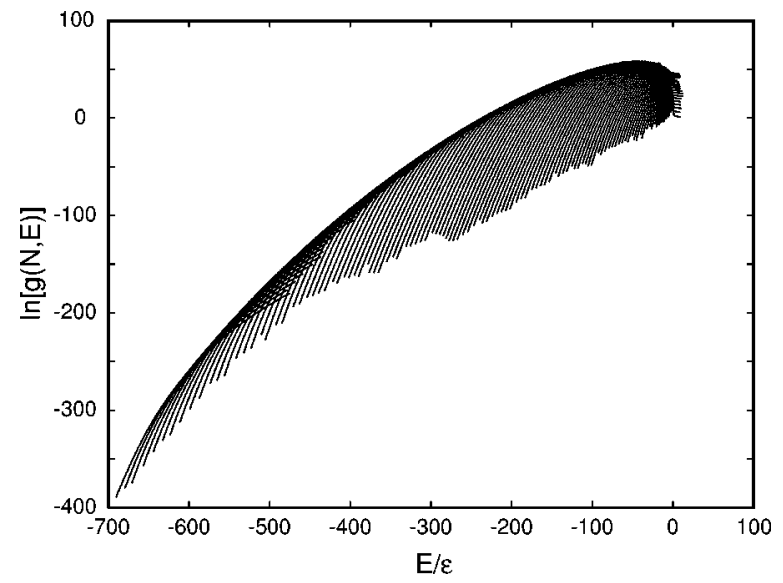

FIG. 1. Two-dimensional density of states of the truncated Lennard-Jones fluid. Different lines correspond to a different number of particles. The number of particles increases monotonically from right to left.

tion simulation. In the scheme described here, there is no need to determine those weights, as they would be determined directly through the course of the simulation.

\section{APPLICATION TO LENNARD-JONES FLUIDS}

The remainder of this article describes results from the application of the DOS method outlined above to the particular case of a truncated Lennard-Jones fluid. This model exhibits most of the main features that one expects to find in most realistic fluids, and offers the advantage that highaccuracy simulation data are available for its thermodynamic properties. The potential energy of interaction between two particles is of the form

$$
U(r)=\left\{\begin{array}{l}
0, \quad r \geqslant r_{\mathrm{c}} \\
4 \epsilon\left[\left(\frac{\sigma}{r}\right)^{12}-\left(\frac{\sigma}{r}\right)^{6}\right], \quad r<r_{\mathrm{c}},
\end{array}\right.
$$

where $r$ is the distance between the particles, and $r_{\mathrm{c}}$ is the cutoff distance. To compare our results to those reported in the literature, we use $r_{\mathrm{c}}=2.5 \sigma$. The box length of the system is set to $L=5 \sigma$.

Before a simulation is conducted, a relevant range of interest for the number of particles and the energy must be specified. In this work, that range is set to be between 0 to 110 for the number of particles, which covers the density range $\rho^{*}=0-0.88$. The range of energy comprises $E / \epsilon$ $=-690$ at one end of the spectrum, and $E / \epsilon=10$ at the other end; this range corresponds to temperatures in the range $0.5<T^{*}<1.5$.

In contrast to a spin lattice, the potential energy of a Lennard-Jones fluid is continuous; a discretization of the energy must therefore be introduced. In this work, an energy bin size of $\epsilon$ is used to construct the density of states and the required histograms. Approximately $5 \times 10^{7}$ Monte Carlo steps were used to generate the complete density of states.

Figure 1 shows the density of states as a function of energy and number of particles. Note that for most $(N, E)$ pairs, the corresponding density of states is less than unity.

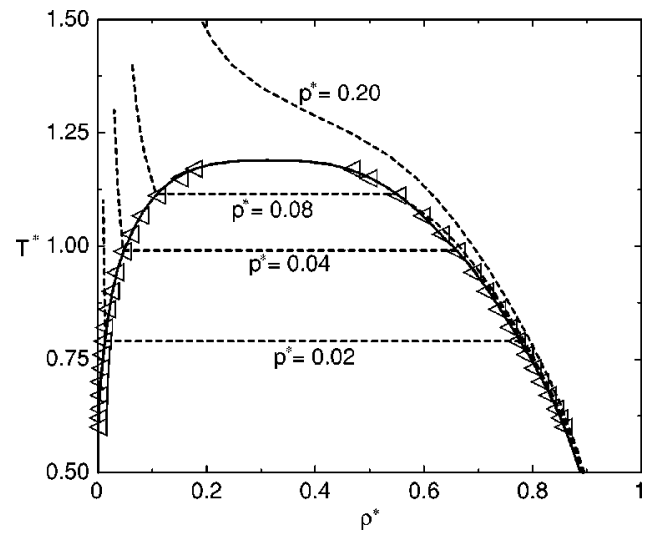

FIG. 2. Phase diagram of the truncated Lennard-Jones fluid. The solid line shows the results of this work; the triangles depict literature data for the same system (Ref. 8). The dashed lines are isobars calculated from the density of states.

This apparently paradoxical feature is actually due to the fact that the de Broglie thermal wavelength is set to unity in our simulations.

Figure 2 shows the phase diagram of the truncated Lennard-Jones fluid. The solid line was calculated from the density of states determined in this work; the triangles show literature results for the same system. ${ }^{8}$ The agreement between the two sets of data is good. The figure shows several isobars, also calculated from the density of states. These isobars are in excellent agreement with pressure calculations from conventional canonical-ensemble simulations (results not shown). The isothermal compressibility of the system, calculated according to Eq. (8), is shown in Fig. 3 as a function of density along several isobars. These results are also consistent with those of conventional simulations. A distinct peak can be observed in the compressibility near $\rho=0.3$. As expected, the peak becomes more pronounced as the pressure approaches the critical pressure of the system (and fluctuations become more prominent).

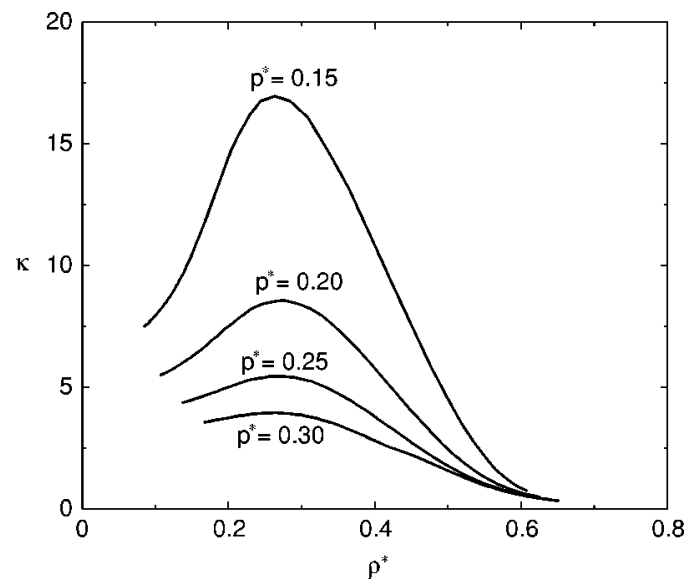

FIG. 3. Compressibility of the truncated Lennard-Jones fluid as a function of density, along various supercritical isobars. 


\section{CONCLUSIONS}

A density of states Monte Carlo method has been presented for simulation of the thermodynamic properties of realistic fluids over wide ranges of density and energy. This method permits calculation of virtually all of the thermodynamic properties of a system, including its phase behavior, as a function of density or temperature; all of this information is generated from a single simulation. Furthermore, by virtue of the way in which trial configurations are generated, states of the system that would otherwise be difficult to sample (e.g., low temperatures or high densities) can be studied efficiently and with remarkable accuracy. An additional benefit of the method is that prior knowledge of the behavior of the fluid (such as approximate location of phase boundaries) is not required; a "blind" simulation is able to sample relevant configurations of the system with little, if any user-supplied guidance.

For the particular case of a truncated Lennard-Jones fluid, it has been shown that the procedure proposed in this work generates thermodynamic information in quantitative agreement with high accuracy literature data for the same fluid. The coexistence curve, vapor pressure, and isothermal compressibility are all in agreement with results of conventional simulation techniques. For this fluid, the entire range of temperature and density considered in this work can be generated in several hours of computer time.

While in this work the DOS Monte Carlo method has only been applied to a simple fluid, our preliminary results for polymeric systems, glasses, and model proteins indicate that the method offers considerable promise for simulation of truly complex fluids.

\section{ACKNOWLEDGMENT}

Financial support provided by the National Science Foundation (CTS-9901430) is gratefully acknowledged.

\footnotetext{
${ }^{1}$ A. A. Lyubartsev, A. A. Martsinovski, S. V. Shevkunov, and P. N. Vorontsov-Velyaminov, J. Chem. Phys. 96, 1776 (1992).

${ }^{2}$ F. A. Escobedo and J. J. de Pablo, J. Chem. Phys. 105, 4391 (1996).

${ }^{3}$ B. Berg and T. Neuhaus, Phys. Lett. B 267, 249 (1991).

${ }^{4}$ C. J. Geyer and E. A. Thompson, J. Am. Stat. Assoc. 90, 909 (1995).

${ }^{5}$ N. Metropolis, W. W. Rosenbluth, M. N. Rosenbluth, A. H. Teller, and E. Teller, J. Chem. Phys. 21, 1087 (1953).

${ }^{6}$ F. Wang and D. P. Landau, Phys. Rev. Lett. 86, 2050 (2001).

${ }^{7}$ A. M. Ferrenberg and R. H. Swendsen, Phys. Rev. Lett. 61, 2635 (1988).

${ }^{8}$ Q. L. Yan and J. J. de Pablo, J. Chem. Phys. 11, 9509 (1999).
} 